\title{
LA RESILIENCIA: UNA MIRADA DESDE LA ENFERMERÍA
}

\author{
RESILIENCE: A VIEW FROM NURSING
}

\author{
JuAna Elena Cortés RecabaL
}

\begin{abstract}
RESUMEN
El objetivo del artículo es demostrar la importancia del concepto de resiliencia para los profesionales de enfermería, presentando una reflexión teórica acerca de las distintas definiciones del concepto y dando a conocer además una aproximación de un modelo de enfermería que se relaciona con el tema, analizando los supuestos y su vinculación con la resiliencia. Finalmente se plantean algunas consideraciones generales, sugerencias y su aplicación a la disciplina de la enfermería.
\end{abstract}

Palabras clave: Resiliencia, enfermería, salud, entorno, persona, modelo.

\begin{abstract}
The objective of this article is to show the importance of the concept of resilience to nursing professionals through the presentation of a theoretical reflection on the different definitions of the concept, also presenting a method for creating a nursing model related to the topic by analyzing the assumptions and associations of resilience. Finally, it raises some general considerations, suggestions and its application to the discipline of nursing.
\end{abstract}

Key words: Resilience, nursing, health, surrounding, person, model.

Fecha recepción: 26/11/09 Fecha aceptación: 14/08/10

\section{INTRODUCCIÓN}

Desde hace algunos años ha comenzado a manejarse el concepto de resiliencia como aquella cualidad de las personas para resistir y rehacerse ante situaciones traumáticas o de pérdida. La resiliencia se ha definido como la capacidad de una persona o grupo para seguir proyectándose en el futuro a pesar de acontecimientos desestabilizadores, condiciones de vida difíciles y de traumas graves. Aunque durante mucho tiempo las respuestas resilientes han sido consideradas como inusuales e incluso patológicas por los exper- tos, la literatura científica actual demuestra de forma contundente que la resiliencia es una respuesta común y su aparición no indica patología, sino un ajuste saludable a la adversidad. Para el profesional de enfermería, el desarrollo de la resiliencia requiere otra forma de mirar la realidad para usar mejor las estrategias de intervención. Más allá de los síntomas y las conductas, esa mirada intenta detectar y movilizar los recursos de las personas, de su entorno, de los servicios y redes sociales con las que cuenta, logrando por un lado una correcta interpretación y evaluación de ésta, que permitirá desarrollar mecanismos de acción preventiva y cambios 
favorables en el estilo de vida.

Promover la resiliencia es un llamado a relacionarse con un individuo en su totalidad, trabajar con un enfoque interdisciplinario, llevando a la práctica el concepto "biopsico-social”. La resiliencia y la prevención de enfermedades y/o patologías individuales y sociales demandan una aproximación dinámica que requiere de un enfoque integral y participativo.

El objetivo del artículo es demostrar la importancia del concepto de resiliencia para los profesionales de enfermería.

\section{CONCEPTUALIZACIONES DE LA RESILIENCIA}

El vocablo resiliencia tiene su origen en el idioma latín, en el término "resilio", que significa volver atrás, volver de un salto, resaltar, rebotar. A lo largo de la historia, el concepto de resiliencia ha tenido varias definiciones.

Para Rutter (1), sirve "para caracterizar aquellas personas que, a pesar de nacer y vivir en situaciones de alto riesgo, se desarrollan psicológicamente sanos y exitosos".

Cyrulnik (2), como uno de los mayores exponentes en el mundo de la teoría y práctica de la resiliencia, la define como "la capacidad de los seres humanos sometidos a los efectos de una adversidad, de superarla e incluso salir fortalecidos de la situación".

Vanistendael (3) habla de una capacidad universal que permite a una persona, grupo o comunidad impedir, disminuir o superar los efectos nocivos de la adversidad.

En tanto Walsh (4) la menciona como la capacidad de una persona para recobrarse de la adversidad fortalecida y dueña de mayores recursos. Se trata de un proceso activo de resistencia, autocorreción y crecimiento como respuesta a las crisis y desafíos de la vida.

Otros autores (2) hablan de historia de adaptaciones exitosas en el individuo que se ha visto expuesto a factores biológicos de riesgo o eventos de vida estresantes.

Cabe destacar la definición que proporciona Grotberg (5) sobre resiliencia: "Capacidad humana universal para hacer frente a las adversidades de la vida, superarlas o incluso ser transformados por ella". Considera la resiliencia como parte del proceso evolutivo y debe ser promovido desde la niñez.

Autores (6) concluyen que determinados factores, como la adquisición de responsabilidades, el altruismo hacia el prójimo como medio de reparación personal, sentirse amado, la fe y búsqueda de filosofía, otorgan sentido a la existencia y permiten al individuo encontrarle cierto sentido a su sufrimiento.

En conclusión, es posible concordar con la literatura reciente sobre resiliencia (5) que sugiere que, a pesar de que es importante trabajar por la promoción de factores resilientes específicos, es crucial avanzar en la investigación y elaboración de teorías que expliquen cómo estos factores específicos interactúan entre sí en la ecología del individuo, permitiendo el proceso de una adaptación resiliente.

Entre las distintas definiciones de resiliencia, la mejor representada es la adoptada por la segunda generación de investigadores como Luthar (7), quienes definen resiliencia como "un proceso dinámico que tiene como resultado la adaptación positiva en contextos de adversidad". Esta definición distingue tres componentes esenciales que deben estar presentes en el concepto de resiliencia:

1. La noción de adversidad, trauma, riesgo, o amenaza al desarrollo humano.

2. La adaptación positiva o superación de la adversidad.

3. El proceso que considera la dinámica entre mecanismos emocionales, cognitivos y socioculturales que influyen sobre el desarrollo humano.

En relación a lo mencionado anterior- 
mente, es importante entender la resiliencia como un proceso de superación de la adversidad y de responsabilidad social y política, ya que ésta puede ser promovida con la participación de todos los actores de la sociedad. De esta forma, la resiliencia permite una nueva epistemología del desarrollo humano, en tanto enfatiza el potencial humano, es específica de cada cultura y hace un llamado a la responsabilidad colectiva. Un enfoque en resiliencia permite que la promoción de la calidad de vida sea una labor colectiva y multidisciplinaria (7).

Existen 8 características personales denominados pilares de la resiliencia (5). Éstos son:

1. Independencia. Se define como la capacidad de establecer límites entre uno mismo y los ambientes adversos; distancia emocional y física.

2. Introspección o capacidad de insight. Es la capacidad de mirarse a sí mismo, detectar y reconocer las propias emociones.

3. Capacidad de relacionarse o interacción. Es la habilidad para establecer lazos íntimos y satisfactorios con otras personas, es crear vínculos.

4. Iniciativa. Es el placer de exigirse y ponerse a prueba en tareas progresivamente más exigentes.

5. Humor. Alude a la capacidad de encontrar lo cómico en la tragedia.

6. Creatividad. Es la capacidad de crear orden, belleza y finalidad a partir del caos y el desorden, es hacer todo a partir de la nada.

7. Moralidad. Es la actividad de una conciencia informada, se refiere a la conciencia moral, a la capacidad de comprometerse con valores y de discriminar entre lo bueno y lo malo.

8. Autoestima consistente. Es la base de los demás pilares y fruto del cuidado afectivo consecuente del niño o adolescente por un adulto significativo.

\section{MODELO DE ENFERMERÍA Y SU RELACIÓN CON LA RESILIENCIA}

El modelo del sistema conductual de Dorothy Johnson (Anexo 1) se centra en cómo el paciente se adapta a la enfermedad y en cómo el estrés presente o potencial puede afectar la capacidad de adaptación. Para Johnson, el objetivo de enfermería es reducir el estrés de tal forma que el paciente pueda avanzar con mayor facilidad a través del proceso de recuperación (8). El modelo de Johnson se centra en las necesidades básicas en relación en los siguientes subsistemas de conductas:

1. Subsistema de afiliación. Es probablemente el más importante, asegura la supervivencia y la seguridad.

2. Subsistema de dependencia. Promueve una conducta de colaboración que exige una respuesta recíproca.

3. Subsistema de ingestión. Tiene que ver con cuándo, cómo, qué, cuánto y en qué condiciones nos alimentamos. Cumple así la amplia función de satisfacer el apetito.

4. Subsistema de eliminación. Tiene que ver con cuándo, cómo y en qué condiciones se produce la eliminación, considera aspectos biológicos, sociales y fisiológicos.

5. Subsistema sexual. Obedece a la doble función de la procreación y la gratificación. 
6. Subsistema de realización. Este subsistema trata de manipular el entorno, controla y domina un aspecto propio o del mundo circundante hasta alcanzar cierto grado de superación.

7. Subsistema agresión/protección. Consiste en proteger y conservar.

Los supuestos principales de esta teoría (8) son:

Enfermería. Es una fuerza externa que actúa para preservar la organización de la conducta del paciente cuando éste se siente sometido a estrés, por medio del fomento de mecanismos reguladores y otros recursos. Como técnica y como ciencia, presta asistencia externa antes y durante la pérdida del equilibrio del sistema y, por lo tanto, requiere un conocimiento del orden, el desorden y el control.

Persona. Concibe a la persona como un sistema conductual con formas de comportamiento marcadas por un modelo, repetitivas e intencionadas, que la vinculan con el entorno.

Johnson (8) presupone también que un sistema conductual es fundamental para el individuo, y cuando alguna fuerza poderosa y una menor resistencia perturban el equilibrio del sistema conductual la integridad del individuo se ve amenazada.

Salud. Estado dinámico fugaz influido por factores biológicos, fisiológicos y sociológicos. La salud se refleja en la organización, interacción, interdependencia e integración de los subsistemas que integran el sistema conductual.

Entorno. Se compone de todos los factores que no forman parte del sistema conductual del individuo pero que influyen en él; algu- nos de ellos pueden ser utilizados por la enfermera a favor de la salud del paciente.

\section{RELACIÓN ENTRE EL MODELO DE ENFERMERÍA Y LA RESILIENCIA}

El objetivo del modelo consiste en mantener y recuperar el equilibrio en el usuario, ayudándole a conseguir un nivel óptimo de funcionamiento, es por esto que se relaciona directamente con el término "resiliencia", que es la capacidad que va adquiriendo la persona para poder enfrentar, satisfactoriamente, los distintos factores de riesgo, y a la vez ser fortalecido como resultado de esa situación.

El sistema humano está constantemente sometido a factores estresantes, los cuales producen un desequilibrio en el individuo. Es aquí donde el profesional de enfermería debe intervenir proporcionando cuidados que lleven al usuario a enfrentar de mejor manera las amenazas tanto internas como externas que se le presentan en diversas ocasiones y así lograr que las personas enfrenten estos factores de manera positiva, lo que los llevará a ser una persona resiliente.

Partiendo de la base que los sistemas son interactivos, interdependientes e integrados, podemos decir que "los cambios en un subsistema producidos por una enfermedad no se pueden comprender del todo sin entender su relación con los cambios producidos en los otros subsistemas". Es así como los principios del modelo del sistema conductual permiten a la enfermera/o valorar la importancia de ofrecer de forma constante protección, nutrición y estímulo para así fomentar la resiliencia en el individuo (9).

Según Johnson (9), las personas son seres activos en constante labor de búsqueda no sólo para adaptarse a su entorno, sino también para alterar dicho entorno y adap- 
tarlo a sus necesidades. Asimismo, considera que el sistema conductual es mas activo que reactivo.

Otros autores (10) hablan que factores de riesgos propios de las enfermedades y lesiones son un enfoque comúnmente utilizado para conocer cuán preparados están las personas para experimentar un problema de salud. La exposición al estrés o a un desafío psíquico es fundamental para definir la resiliencia en los seres humanos.

\section{CONSIDERACIONES FINALES}

La resiliencia no es un estado definido y estable, es más bien un camino de crecimiento. La resiliencia no se construye sola, sino gracias a los fuertes vínculos de afecto que se han tejido a lo largo de toda la vida.

Cambiar de mirada, tener una mirada positiva sobre las personas y sus capacidades implica, por ejemplo, incluir sistemáticamente en la anamnesis las preguntas sobre los aspectos positivos del desarrollo y la salud.

Para el profesional (11) el desarrollo de la resiliencia requiere otra forma de mirar la realidad para usar mejor las estrategias de intervención. Más allá de los síntomas y las conductas, esa mirada intenta detectar y movilizar los recursos de las personas, de su entorno, de los servicios y las redes sociales.

Finalmente, se sugiere: dar a conocer y difundir el concepto de resiliencia entre los profesionales del área de la salud; estudiar la resiliencia como un proceso, lo que plantea nuevos desafíos metodológicos; relacionar los modelos de enfermería sobre la resiliencia con el quehacer profesional y reconocer los aspectos positivos de los usuarios que evidencien una buena capacidad de resiliencia.

\section{REFERENCIAS}

1. Kotliarenco M, Caceres I, Fontecilla M. Estado del arte en resiliencia. [Internet]. 1997. Hallado en: http://www.resiliencia. cl/resilien.htm. [acceso 25 junio 2006].

2. Melillo A. Sobre resiliencia: El pensamiento de Boris Cyrulnik. Perspectivas Sistemáticas. [Internet]. 2005. Hallado en: http://www.redsistemica.com.ar/melillo.htm. [acceso el 22 de agosto 2010].

3. Manciaux M, Vanistendael S (comp). La resiliencia en lo cotidiano. La resiliencia: resistir y rehacerse. Barcelona: Gedisa; 2003. p. 17-21-23-103-105.

4. Walsh F. Resiliencia familiar. Buenos Aires: Amorrortu; 2004. p. 396-397.

5. Grotberg E. Descubriendo las propias fortalezas. Buenos Aires: Paidós; 2001. p. 23-25.

6. Melillo A, Suárez N, Rodríguez D. (comp). Resiliencia y subjetividad: Los ciclos de la vida. Buenos Aires: Paidós; 2006. p. 91-101.

7. Melillo A, Suárez N, Ojeda E (comp). Resiliencia descubriendo las propias fortalezas. Buenos Aires: Paidós; 2001. p. 35-41.

8. Potter PA, Perry AG. Fundamentos de enfermería: teoría y práctica. $3^{a}$ ed. Madrid, España: Mosby/Doyma; 1996. p. 1267.

9. Marriner-Tomey A, Alligood MR. Modelos y teoría en enfermería. $4^{\mathrm{a}}$ ed. Madrid, España: Hartcourt/Brace; 1999. p. 229-231.

10. Grotberg E (comp). La resiliencia en el mundo de hoy. Cómo superar las adversidades. Barcelona: Gedisa; 2006. p. 216.

11. Manciaux M, Vanistendael S (comp). La resiliencia: estado de la cuestión. La resiliencia: resistir y rehacerse. Barcelona: Gedisa; 2003. p. 23. 


\section{Anexo 1}

Diagrama representativo del modelo teórico del sistema conductual de Dorothy Johnson

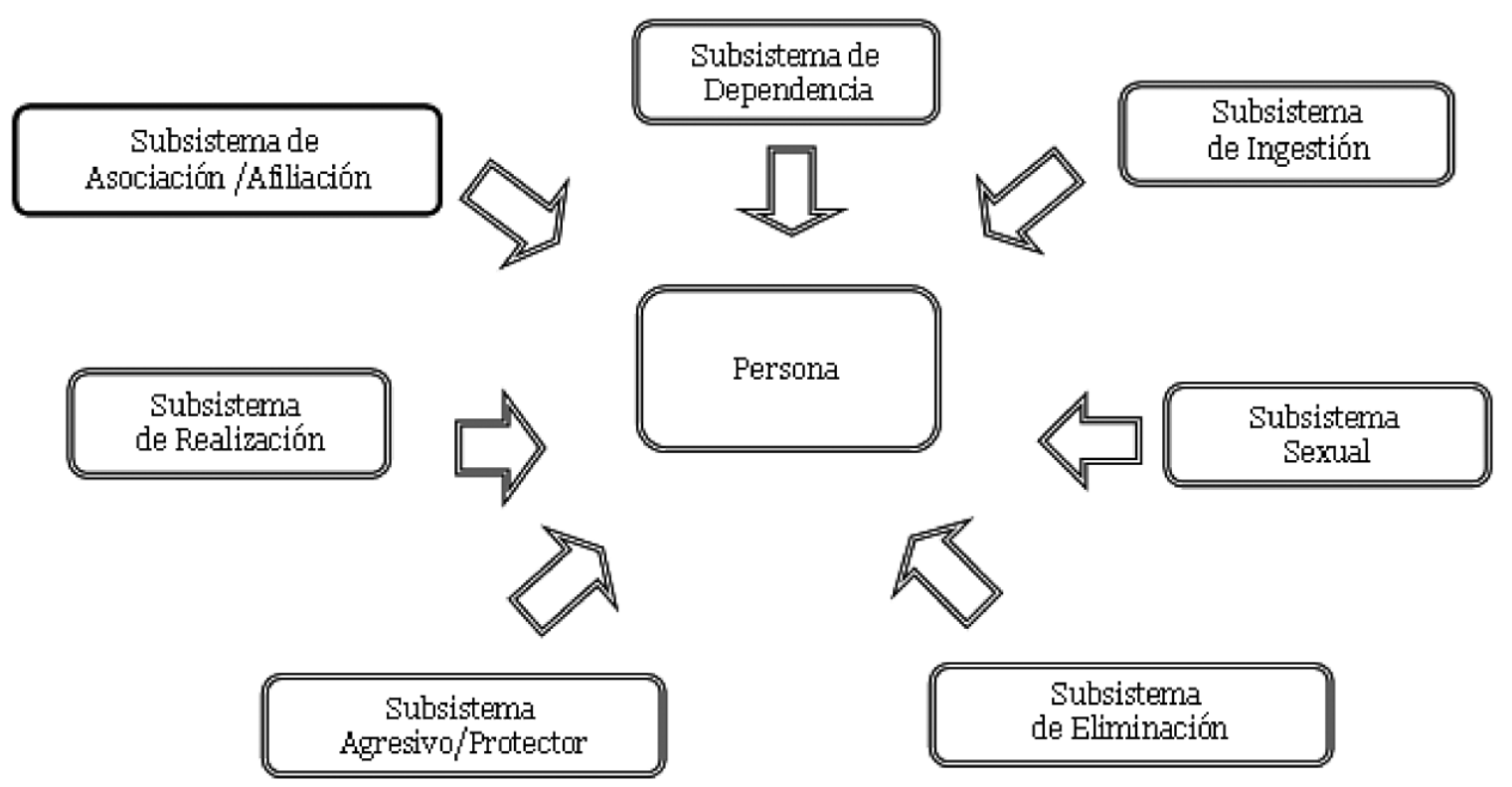

